\title{
Virtual Prototyping of a Catheter Transducer Array for Internal Hepatic Sonoporation
}

\author{
Alexandru Corneliu Moldovan, Jerzy Dziewierz, Anthony Gachagan \\ Centre for Ultrasonic Engineering \\ University of Strathclyde \\ Glasgow, United Kingdom \\ alexandru.moldovan@strath.ac.uk
}

\author{
Sandy Cochran, Holly Lay \\ School of Engineering \\ University of Glasgow \\ Glasgow, United Kingdom
}

\begin{abstract}
Sonoporation for targeted cancer chemotherapy in the liver with an external ultrasonic source is hampered by the ribs and fat surrounding the organ. Current therapies rely on array beam forming with sequential triggering of elements to spare the ribs. However, the peak negative pressures (PNPs) achieved in hepatic tumors are rather low and there is risk associated with high power ultrasound incident on the ribs. Here, we focus on the development of a 1-3 connectivity piezocomposite linear ultrasonic array that can be incorporated in an $11 \mathrm{Fr}$ catheter to perform sonoporation of the liver from within a larger blood vessel. Issues encountered in the transducer design are mostly caused by the reduced dimensions of the catheter, which are inimical to the low frequency and high power therapeutic requirements. Thus, our approach to produce a transducer design is through a series of parametric sweeps of array parameters using finite element analysis. The output parameters of interest are PNP and beam forming to steer at more than $45^{\circ}$ from normal. Two frequencies are considered: 1.5 MHz, set by the maximum physical transducer thickness, and 3.0 $\mathrm{MHz}$, corresponding to the frequency of resonance of the contrast agents to carry the drugs. Piezoelectric materials of interest are ceramic (PZT-5H) and single crystal (PMN-29\%PT, $26 \%$ PIN-PMN-32\%PT). The parametric sweeps indicate that fewer pillars laterally per element, higher volume fraction $(>0.68)$ and higher pillar aspect ratio $(>0.42)$ achieve the lowest PNP in the load.
\end{abstract}

Keywords - medical ultrasound; sonoporation; targeted drug delivery; ultrasonic linear array.

\section{Clinical BackgRound AND Motivation}

Sonoporation is a non-invasive acoustic technique that relies on several ultrasound-induced phenomena including cavitation, shock waves, acoustic streaming and jetting to temporary permeabilise the cellular membrane to the larger drug molecules [1]. The efficiency of sonoporation is increased by injecting gas filled contrast agents (GCA) whose presence lowers the cavitation threshold and whose mechanical oscillations perturb the integrity of cellular membranes [2].

A major limitation of this therapy is posed by the presence of media with significantly different acoustic properties than the surrounding tissue in the acoustic path between the source and the target. One example is the ribs, which absorb acoustic energy, scatter the acoustic waves, and cause reflections and interference between them. During high power ultrasound treatment, significant heat accumulation at the transducer - skin interface and inside the ribs has been reported [3]. Current solutions place the transducer below the rib cage or use beam steering techniques to avoid the ribs. Even so, the intensity levels achieved in the target tissue are reduced [3]. Other

Mr. Moldovan's PhD Studentship is funded through an Engineering and Physical Sciences Research Council (EPSRC) Doctoral Training Grant (EP/N509760/1) causes can be nonlinear wave propagation and attenuation due to the distance between the external transducer and the liver [4]. Furthermore, these methods pose the risk of harming the patient due to acoustic energy absorbed by the ribs during therapy. Any dysfunctions in the level of bilirubin or increased fat layer surrounding the liver lead to increased heat deposition and are contraindications in high power ultrasound therapies [5].

\section{AIM OF STUDY}

This paper presents the design procedure for a linear phased array to fit into an 11 Fr catheter, Fig. 1. With a transducer operating from within a larger hepatic blood vessel, most of the shortcomings because of obstacles in the acoustic path and large propagation distances can be solved. Furthermore, the catheter can be fitted with a microbubble (MB) feeder tube to deliver the drug and contrast agents locally near the tumour.

\section{Catheter Lateral View}

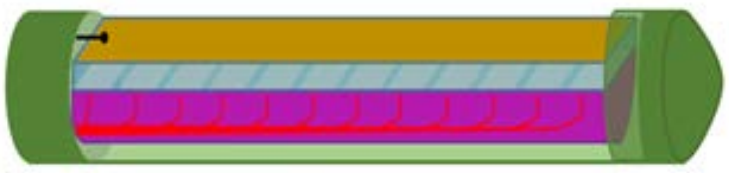

Catheter Cross Section View

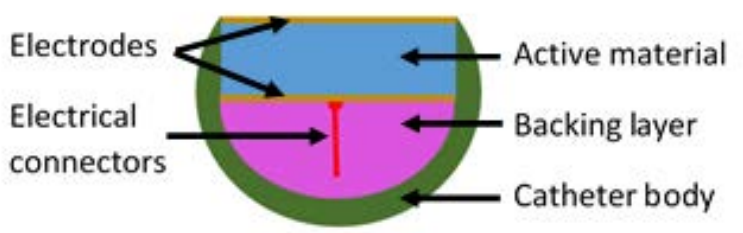

Fig. 1. Proposed ultrasonic catheter linear array for sonoporation.

Catheter implementation of a linear array imposes serious restrictions on the element size as well as on its thickness, which is inversely related to the resonance frequency of the transducer. Sonoporation efficiency is dependent on both frequency and peak negative pressure (PNP), with studies showing that satisfactory therapeutical results are obtained for insonation frequencies below $5 \mathrm{MHz}$ [6]. Furthermore, cavitation, as an important mechanism for sonoporation is more difficult to track at higher frequencies due to the reduced bubble resonance radius and increased tissue heating through absorption. The reduced transducer element surface leads to high beam divergence which affects the focusing power of the array and the pressure swing it can deliver to the target. To design a transducer for efficient sonoporation, a series of parameter sweeps is performed to obtain the configuration that 
offers the best results in terms of PNP and beam forming capabilities, with dimensions constrained to fit inside the catheter.

\section{TECHNICAL BACKGROUND}

The active piezoelectric material in the transducer is 1-3 composite chosen for its advantages over bulk ceramic: better acoustic matching with the target tissue, reduced thickness and higher coupling coefficient. The array is designed to be manufactured using the conventional dice and fill method and three piezoelectric materials are investigated: PZT-5H, PMN$29 \% \mathrm{PT}$ and $26 \% \mathrm{PIN}-\mathrm{PMN}-32 \% \mathrm{PT}$. The kerfs are filled with Epofix (Struers, UK). The backing layer is made of microballoon filled epoxy moulded in the shape of the catheter and a matching layer is not included in the design because of the high power transmission. Two resonance frequencies are investigated: $1.5 \mathrm{MHz}$, set by the maximum transducer thickness that can fit in the catheter, and $3.0 \mathrm{MHz}$, corresponding to the resonance frequency of the contrast agents used to promote sonoporation.

The modelled transducer is required to focus up to $25 \mathrm{~mm}$ in depth and to steer the ultrasound beam at least $45^{\circ}$ from normal. This is estimated to allow insonation of the entire volume of a typical tumour without movement of the catheter, thereby reducing the risks associated with the procedure. Furthermore, the focal spot size can be adjusted by excitation signal apodisation which can increase the effectiveness of sonoporation.

Finite element analysis (FEA) is a computational method in which a modelled space is divided into small cells - the finite elements - with solutions of the governing equations determined at the nodes of the cells. This method allows simulation of complex 2D and 3D geometries through the production of unique, convergent results; in contrast, 1D analytical models carry the risk of overly complex and diverging solutions. Because of recent advances in computer power and consequently the number of finite elements that can be modelled, the accuracy of FEM can be increased while keeping the computation times reasonable. Results obtained with FEM can well predict a transducer's behaviour and reduce the time for further design and optimisation [7].

\section{METHODOLOGY}

A graphical user interface program was designed in MATLAB (The MathWorks, Cambs, UK) to calculate the full set of required array parameters from a number of base parameters given by size restrictions, array performance and manufacturing considerations.

The input parameters are: electrical resonance frequency, approximate array length and elevation, piezoelectric and polymer materials, kerf width $(\mathrm{KW})$ interval, and number of array elements. The swept parameters are volume fraction (VF) and pillar aspect ratio (PAR). The longitudinal acoustic velocity, the electromechanical coupling coefficient and the composite thickness are calculated using an analytical method [8] and the first and second spurious modes are also predicted in this way [9]. Other calculated parameters are: the exact $\mathrm{KW}$, pillar pitch (PP), number of pillars per array element in the array length $(\mathrm{X})$ and elevation $(\mathrm{Y})$ directions, electrode spacing as a function of wavelength in the load and composite acoustic impedance.

The first parameter swept is VF and the reported model output is PNP. VF is related to the pillar width (PW), PP and KW through Equation (1):

$$
V F=\left(\frac{P W}{P P}\right)^{2}=\left(\frac{P W}{P W+K W}\right)^{2}
$$

PAR is also related to $\mathrm{VF}$ through $\mathrm{PW}$, which is the common parameter. PAR, KW, PP and X and Y pillar numbers are determined so that the element $\mathrm{X}$ and $\mathrm{Y}$ dimensions are within $\pm 10 \%$ bounds of the desired setting. This is required in order to have a fair comparison between the swept models, i.e. so that the radiating area does not vary greatly between models, and to ensure the array fits inside the catheter and has efficient beam steering capabilities.

For the $1.5 \mathrm{MHz}$ array, the number of array elements is fixed at 24, for a transducer length of $11.7 \mathrm{~mm}$ and for the $3.0 \mathrm{MHz}$ case, 36 elements are included in the array giving a length of $8.95 \mathrm{~mm}$. The numbers of array elements were chosen so that the transducer length does not impair insertion in the liver whilst providing an active aperture large enough for efficient focusing. The elevation is set to $2.4 \mathrm{~mm}$ in all cases because of the catheter diameter restriction. The transducer is coupled to a water load and absorbing boundaries are added to the finite element model (FEM). The depth of the water is set to be larger than 2 wavelengths so that data for extrapolation of the field can be achieved without any influence from the boundaries.

Delay laws are applied to the array elements to focus the transducer at a focal distance of $20 \mathrm{~mm}$. This option has proven better than simulating just one element and measuring its PNP at the near field because it takes into consideration the entire array behaviour. A $100 \mathrm{~V}_{\text {peak }}, 10$-cycle sinusoidal signal is used excite the elements and the model run time is calculated so that the mechanical wave generated by the transducer has time to propagate through the entire load medium. No damping circuit is connected to the transducer model because the ring down time is not important.

After running the PZFlex (OnScale Inc, Cupertino, CA) FEM model, time response extrapolations are executed at points along the line perpendicular to the centre of the array towards the water load. The minimum value of the pressure as a function of time at each point represents the PNP at that point. This allows PNP to be plotted as a function of distance from the array. The focal point of the array is determined through a simple optimisation algorithm and the pressure value at that point is associated with the lowest value of PNP attainable with the current array configuration. Extrapolation of time functions from FEM data is better than simpler analytical methods because, rather than a normalized value, it provides a value of pressure magnitude which can be used as the target parameter for the parametric sweep.

Extrapolations of the pressure along multiple lines, as described above are executed along the array length and elevation to form two 2-D plots of the beam profile and thus offer insight into the pressure distribution in the load medium. 
A similar VF sweep is executed afterwards, but the output parameters tracked are electrical impedance and mode shapes at various resonance frequencies. The excitation signal is set to a half cycle sinusoid of amplitude 1 Vpeak and the simulation time is increased so that the transducer rings down. A series damping resistor is added to the circuit and the value is calculated to match the impedance of the element at electrical resonance to reduce the ring down time. The electrical impedance is verified in two cases: firstly a single element is modelled with symmetry boundaries to evaluate its electrical resonant frequency as part of an infinite composite. In the second case, the entire array is modelled with the central element excited and all other elements left open circuit. This configuration shows the mode propagation in the composite structure and can be used to explain the PNP variation at different volume fractions.

Finally, a PAR sweep is performed to evaluate its influence over the attainable PNP. The VF is kept constant at the optimised value calculated beforehand, while $\mathrm{KW}, \mathrm{PW}$ and $\mathrm{X}$ and $\mathrm{Y}$ pillar numbers are changed so that the correct ratios are maintained.

\section{RESULTS AND DISCUSSION}

The VF sweep was performed for $0.4 \leqslant \mathrm{VF} \leqslant 0.8$ for all cases. The results for $\mathrm{PMN}-29 \% \mathrm{PT}$ at $1.5 \mathrm{MHz}$ are presented in Fig. 2. The lowest PNP is for $\mathrm{VF}=0.74$. Table I presents the relation between VF, PAR, and X and Y number of pillars per element.

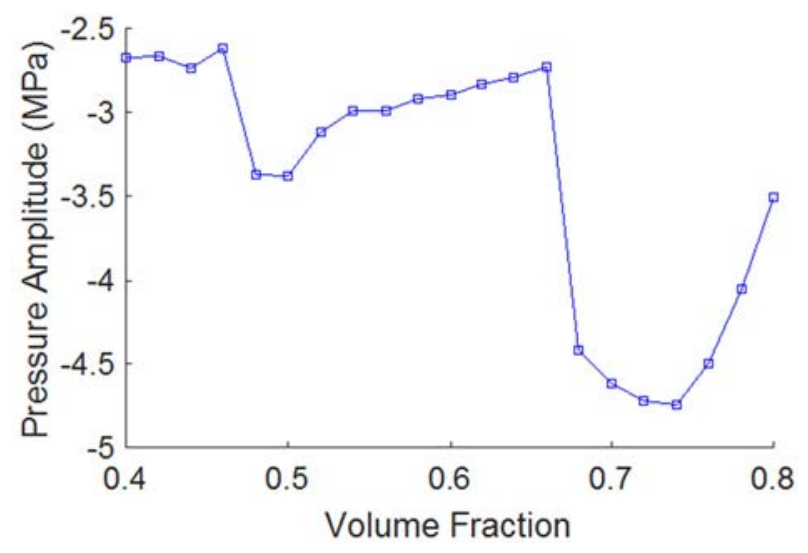

Fig. 2. Peak negative pressure as a function of VF for PMN-29\%PT.

TABLE I. PMN-29\%PT VOLUME FRACTION SWEEP OUTCOMES

\begin{tabular}{|l|c|c|c|c|c|}
\hline $\begin{array}{l}\text { VF } \\
(\%)\end{array}$ & $\begin{array}{c}\text { X } \\
\text { Pillars }\end{array}$ & $\begin{array}{c}\text { Y } \\
\text { Pillars }\end{array}$ & PAR & $\begin{array}{c}\text { Worst PNP } \\
(\mathrm{MPa})\end{array}$ & $\begin{array}{c}\text { Best PNP } \\
(\mathrm{MPa})\end{array}$ \\
\hline $40-46$ & 3 & 15 & $0.15-0.16$ & -2.62 & -2.73 \\
\hline $48-66$ & 2 & 10 & $0.25-0.28$ & -2.73 & -3.38 \\
\hline $68-80$ & 1 & 5 & $0.58-0.59$ & -3.51 & -4.75 \\
\hline
\end{tabular}

This data indicates that configurations with higher values of VF and PAR that have one pillar per element in the $\mathrm{X}$ direction and five in the Y direction achieve the lowest PNPs. The downside of higher PAR is the close proximity of the first spurious mode and the mechanical resonance frequency of the transducer. However, this issue is not important in the case of a therapeutic transducer which is designed to operate solely in transmission mode as the electrical resonance frequency is well decoupled from the spurious modes. Fig. 3 shows the comparison in impedance amplitude between the three most significant cases modelled as infinite composite plates. The traces are close to $1.5 \mathrm{MHz}$ electrical resonance frequency and are not affected by unwanted modes near the electrical resonance frequency.

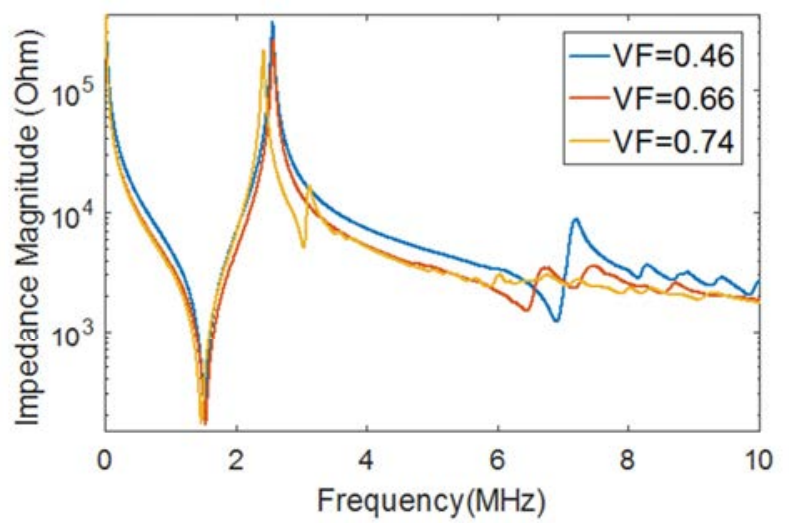

Fig. 3. Impedance magnitude response of an infinite composite plate at three different volume fractions

The impedance magnitude of a single excited element from the entire array, Fig. 4, illustrates that the lateral reflections from the sides of the composite induce a lateral mode that affects the electrical resonance frequency in the cases of lower volume fractions and higher number of pillars per element.

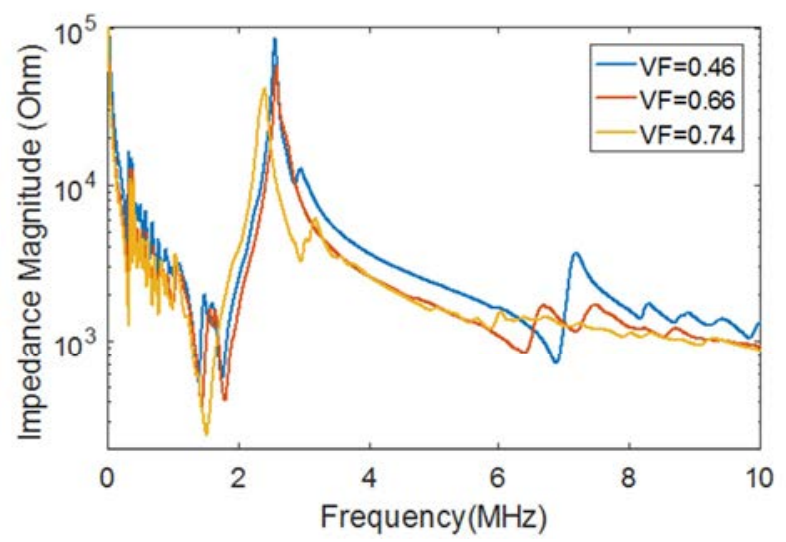

Fig. 4. Impedance magnitude response of the central array element in full array configuration, all other elements open circuit

This result was further investigated by evaluating the mode shapes of the transducers at the peaks and troughs of the lateral modes for the transducers with higher numbers of pillars. This showed evidence that inter-pillar modes are created in the composite lattice and that the surface of the transducer oscillates with node-antinode behaviour for the lower volume fractions, which results in lower net displacements and leads to reduced efficiency. More uniform pillar vibration is evident in the case of higher volume fractions and larger PPs. A possible 
explanation is that a higher concentration of crystal material increases the stiffness of the composite.

In addition, modelling a 2-2 composite configuration with all other parameters identical to the 1-3 configuration gives a PNP at the focus point 2.3x smaller in amplitude than the 1-3 case, which proves the increased efficiency of the 1-3 composite for this particular application.

The 2D beam contour plot of the transducer shows a 1.9 $\mathrm{mm}$ wide beam in the $\mathrm{X}$ direction (Fig. 5) and $5 \mathrm{~mm}$ wide in the $\mathrm{Y}$ direction. The results are consistent with analytical approximations that use Huygens's principle to determine the pressure distribution in the load.

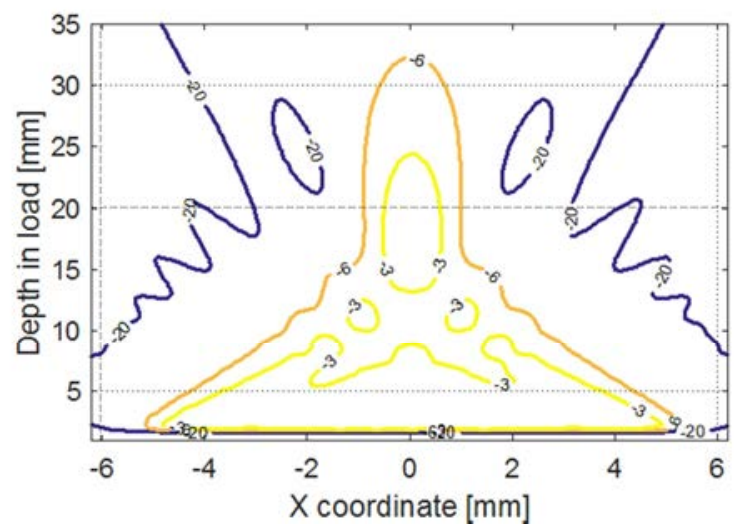

Fig. 5. Beam contour plot for PMN-29\%PT at 1.5 MHz: 2D cross section, $\mathrm{dB}$ contour, along array length

The procedure detailed above has been applied for the remaining transducer cases and the data is collated in Table II.

TABLE II. TRANSDUCER DESIGN PARAMETERS FOR ALL MODELLED TRANSDUCERS

\begin{tabular}{|c|c|c|c|c|c|c|}
\hline $\begin{array}{c}\text { Piezoelectric } \\
\text { Material }\end{array}$ & $\begin{array}{c}\text { FE } \\
(\mathrm{MHz})\end{array}$ & $\begin{array}{c}\text { VF } \\
(\%)\end{array}$ & AR(\%) & $\begin{array}{c}\text { Kerf } \\
(\mu \mathrm{m})\end{array}$ & $\begin{array}{c}\text { Thk } \\
(\mu \mathrm{m})\end{array}$ & $\begin{array}{c}\text { PNP } \\
(\mathrm{MPA})\end{array}$ \\
\hline \multirow{2}{*}{ PZT-5H } & 1.5 & 70 & 42.0 & 80 & 983 & -3.92 \\
\cline { 2 - 7 } & 3.0 & 68 & 42.1 & 44 & 489 & -11.67 \\
\hline \multirow{2}{*}{$\begin{array}{c}\text { PMN- } \\
29 \% \mathrm{PT}\end{array}$} & 1.5 & 74 & 58.1 & 71 & 725 & -4.75 \\
\cline { 2 - 7 } & 3.0 & 74 & 59.3 & 36 & 363 & -17.29 \\
\hline $\begin{array}{c}26 \% \mathrm{PIN}- \\
\text { PMN- } \\
32 \% \mathrm{PT}\end{array}$ & 1.5 & 70 & 56.7 & 80 & 723 & -4.45 \\
\cline { 2 - 7 } & 3.0 & 72 & 58.2 & 38 & 364 & -15.78 \\
\hline
\end{tabular}

The PAR sweep confirmed that slight modifications in PAR did not benefit the transducer performance in terms of PNP. Only in the case of two notable changes led to a decrease of PNP (Table III), but at the cost of increased inter-element spacing, to a level which is not acceptable for efficient beam steering.

TABLE III. NotABLE RESUltS OF PAR SWEEP

\begin{tabular}{|c|c|c|c|c|c|}
\hline $\begin{array}{c}\text { Piezoelectric } \\
\text { Material }\end{array}$ & $\begin{array}{c}\text { FE } \\
(\mathrm{MHz})\end{array}$ & $\begin{array}{c}\text { Old } \\
\text { AR } \\
(\%)\end{array}$ & $\begin{array}{c}\text { New } \\
\text { AR } \\
(\%)\end{array}$ & $\begin{array}{c}\text { New } \\
\text { Spacing } \\
(\lambda)\end{array}$ & $\begin{array}{c}\text { PNP } \\
\text { decrease } \\
(\%)\end{array}$ \\
\hline PZT-5H & 3.0 & 42.1 & 31.4 & 0.75 & 7.8 \\
\hline PMN-29\%PT & 1.5 & 58.1 & 60.4 & 0.6 & 2.6 \\
\hline
\end{tabular}

\section{SUMMARY AND CONCLUSIONS}

This work demonstrates the feasibility of a catheter linear array for therapeutic ultrasound. It also shows the potential value of running a parametric sweep of VF based on FEM at an early stage in the development of 1-3 connectivity piezocomposite ultrasound arrays. Running a follow-up AR sweep may also show improvements in performance, though in the present situation, it demonstrated improved PNP in just two cases. However, the increased element spacing was too large for these to be considered further.

The piezoelectric material that achieved the highest performance in terms of PNP is PMN-29\%PT; this is attributed to its higher coupling coefficient than for the other two materials. Careful composite design also achieved suppression of inter-pillar modes that affect the net displacement of the transducer and thus achieves the desired performance of a low PNP in the load media. Overall, a larger element pitch and VF provide better performance.

Further research will involve manufacturing and characterization of the transducer designs presented in Table II. Finally, the arrays will be tested for cavitation efficiency in water containing ultrasound contrast agents.

\section{REFERENCES}

[1] S. R. Sirsi and M. A. Borden, "Advances in Ultrasound Mediated Gene Therapy Using Microbubble Contrast Agents," Theranostics, vol. 2, no. 12, pp. 1208-1222, 2012.

[2] C. Huang, H. Zhang, and R. Bai, "Advances in ultrasound-targeted microbubble-mediated gene therapy for liver fibrosis," Acta Pharmaceutica Sinica. B, vol. 7, no. 4, pp. 447-452, Jul. 2017.

[3] S. Bobkova, L. Gavrilov, V. Khokhlova, A. Shaw, and J. Hand, "Focusing of high intensity ultrasound through the rib cage using a therapeutic random phased array," Ultrasound Med Biol, vol. 36, no. 6, pp. 888-906, Jun. 2010

[4] J.-L. Li, X.-Z. Liu, D. Zhang, and X.-F. Gong, "Influence of Ribs on the Nonlinear Sound Field of Therapeutic Ultrasound," Ultrasound in Medicine \& Biology, vol. 33, no. 9, pp. 1413-1420, Sep. 2007.

[5] T. T. Cheung et al., "High-intensity focused ultrasound ablation: An effective bridging therapy for hepatocellular carcinoma patients," World J Gastroenterol, vol. 19, no. 20, pp. 3083-3089, May 2013.

[6] J. P. Kilroy, A. V. Patil, J. J. Rychak, and J. A. Hossack, “An IVUS Transducer for Microbubble Therapies," IEEE Trans Ultrason Ferroelectr Freq Control, vol. 61, no. 3, pp. 441-449, Mar. 2014.

[7] N. N. Abboud, G. L. Wojcik, D. K. Vaughan, J. Mould, D. J. Powell, and L. Nikodym, "Finite Element Modeling for Ultrasonic Transducers (Preprint)," WEIDLINGER ASSOCIATES NEW YORK, 1998.

[8] W. A. Smith and B. A. Auld, "Modeling 1-3 composite piezoelectrics: thickness-mode oscillations," IEEE transactions on ultrasonics, ferroelectrics, and frequency control, vol. 38, no. 1, pp. 40-47, 1991.

[9] P. Reynolds, J. Hyslop, and G. Hayward, "Analysis of spurious resonances in single and multi-element piezocomposite ultrasonic transducers," in IEEE Symposium on Ultrasonics, vol.2., pp. 1650-1653, 2003 